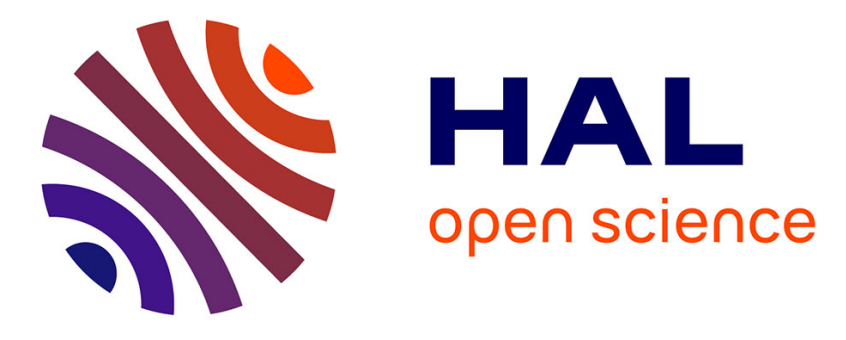

\title{
Characterization of renal tumours based on Raman spectra classification
}

Julien Fleureau, Karim Bensalah, Denis Rolland, Olivier Lavastre, Nathalie

Rioux-Leclercq, François Guillé, Jean-Jacques Patard, Lotfi Senhadji, Renaud de Crevoisier

\section{To cite this version:}

Julien Fleureau, Karim Bensalah, Denis Rolland, Olivier Lavastre, Nathalie Rioux-Leclercq, et al.. Characterization of renal tumours based on Raman spectra classification. Expert Systems with Applications, 2011, 38 (11), pp.14301-14306. 10.1016/j.eswa.2011.05.092 . hal-00907509

\section{HAL Id: hal-00907509 https://hal.science/hal-00907509}

Submitted on 6 Dec 2013

HAL is a multi-disciplinary open access archive for the deposit and dissemination of scientific research documents, whether they are published or not. The documents may come from teaching and research institutions in France or abroad, or from public or private research centers.
L'archive ouverte pluridisciplinaire HAL, est destinée au dépôt et à la diffusion de documents scientifiques de niveau recherche, publiés ou non, émanant des établissements d'enseignement et de recherche français ou étrangers, des laboratoires publics ou privés. 


\title{
Characterization of renal tumours based on Raman spectra classification
}

\author{
Julien Fleureau ${ }^{1,2}$, Karim Bensalah ${ }^{1,3}$, Denis Rolland ${ }^{4}, 5$, \\ Olivier Lavastre ${ }^{4}, 5$, Nathalie Rioux-Leclerq ${ }^{3}$, François Guillé ${ }^{3}$, \\ Jean-Jacques Patard ${ }^{3}$, Lofti Senhadji ${ }^{1,2}$, Renaud de Crevoisier ${ }^{1}, 3$ \\ ${ }^{1}$ Inserm, UMR 642, Rennes, F-35000, France \\ ${ }^{2}$ Université de Rennes 1, LTSI, Rennes, F-35000, France \\ ${ }^{3}$ Université de Rennes 1, Institute of Oncology, Rennes, F-35000, France \\ ${ }^{4}$ Université de Rennes 1, Department of biochemistry, Rennes, F-35000, France \\ ${ }^{5}$ CNRS, UMR 6226, Rennes, F-35000, France \\ For correspondence : Lotfi Senhadji \\ Tel : (33) - 2232355 77, E-Mail : lotfi.senhadji@univ-rennes1.fr
}

\begin{abstract}
In this study, we propose to evaluate the potential of Raman spectroscopy (RS) to assess renal tumours at surgery. Different classes of raman renal spectra acquired during a 5 months clinical protocol are discriminated using Support Vector Machines classifiers. The influence on the classification scores of various preprocessing steps generally involved in RS are also investigated and evaluated in the particular context of renal tumour characterization. Encouraging results show the interest of RS to evaluate kidney cancer and suggest the potential of this technique as a surgical assistance during partial nephrectomy.
\end{abstract}

Keywords: Raman spectroscopy, Cancer, Classification, SVM, Wavelets. 


\section{Introduction}

The natural course of cancer is hardly predictable. This is particularly true for renal cell carcinoma (RCC) where $40 \%$ of affected individuals will die from their disease [1]. A better understanding of molecular pathways has led to the development of multiple targeted therapies with significant clinical benefits [2]. However, response to treatment is only seen in a minority of patients and side effects are frequent and cumbersome. In an effort to better appraise the evolution of RCC in a specific patient, a prognostic role has been suggested for various biomarkers, mostly genes and proteins [3]. Some of these biomarkers have been integrated to predictive models but since they hold many imperfections, they are not used in clinical practice. There is therefore a clear need for new predictive tools and/or different conceptual approaches to address RCC, and more generally cancer [4, 5].

In this direction, several publications have recently presented optical spectroscopy techniques to assess renal tumours. Parekh et al used a combination of reflective and fluorescent spectroscopy, and noted that optical characteristics could differentiate between benign and malignant renal tissues [6]. The team from the University of Texas Southwestern University successively showed that optical reflectance spectroscopy could accurately discriminate normal from tumoural renal tissue [7] and benign from malignant renal tumours at surgery [8].

Another optical spectroscopy approach that is not used, to our knowledge, to evaluate kidney cancer, is the Raman Spectroscopy (RS). Unlike reflectance or fluorescent spectroscopies, RS is based on the molecular inelastic scattering of light [10]: when photons interact with a molecule, the 
transmission of their energy raises the molecule's vibrational state. When the molecule returns to its background level, a photon is emitted at a different wavelength from the incident light. This change in wavelength is known as the Raman effect or Raman shift. All Raman shifts generated by the interaction of light with tissue provide a Raman spectrum that is directly related to the molecular composition of the tissue.

Promising results have been reported with RS in the evaluation of urological tumours. RS has been shown to identify in vitro malignant changes in the urothelium [13] and prostate biopsy cores [12]. In another study, RS could recognize with high accuracy benign prostatic tissue (benign prostatic hyperplasia and prostatitis) from prostate cancer [9]. Nevertheless, RS interest to evaluate kidney cancer still have not been studied until now to our knowledge.

In this study, we therefore propose to investigate the potential of RS to specifically evaluate normal and tumoural renal tissue on surgically removed specimen. More precisely, the discriminant potential of RS will be hereafter shown in different situations of renal raman spectra classification that may have a surgical interest.

A brief description of the data acquisition protocol and an overview of the different groups of renal spectra that should be discriminated are thus first presented in section 2. The global methodology of classification is then described in section 3. Finally, classification scores are presented for various scenarios in section 4 where the interest of RS to evaluate kidney cancer is also shown and discussed. 


\section{Data Acquisition}

\subsection{Specimens}

After approval by our institutional review board, Raman spectra were prospectively collected in consecutive kidney specimen removed due to suspicion of cancer. Between June and November 2009, 43 operations were performed, including 31 radical nephrectomies and 12 partial nephrectomies. The surgery was completed through an open access in 22 patients and laparoscopically in 21 patients. Seven patients were excluded from the study based on final histology results: four with urothelial carcinoma, one with liposarcoma, one with a multicystic non malignant lesion and one with inflammatory cyst. Immediately after extraction, the specimen were stored on ice and transferred to the pathology department. Raman spectra were acquired within 15 minutes after extraction.

\subsection{Instrumentation}

A Raman spectroscopic system was used to acquire immediate Raman spectra from kidney specimen removed at surgery. The system comprised three components: 1) a high-powered near-infrared laser (Invictus NIR laser, Kaiser Optical Systems, Ann Arbor, Michigan, USA) that minimizes fluorescence, 2) a fibre optics MR probe with a 10x non-contact objective, and 3) a RXN1 Raman analyzer (Kaiser Optical Systems, Ann Arbor, Michigan, USA) to receive reflected and scattered light.

\subsection{Raman measurements}

After longitudinal section of the specimen, Raman spectra were acquired by placing the MR probe at several standardized locations on the surface and 
on the section of normal and tumoural tissue. Four Raman measures were made: two inside the tumour section and two on the normal parenchyma section. Two spectra were recorded on each spot. The laser beam was set at a power of $50 \mathrm{~mW}$ and a wavelength of $785 \mathrm{~nm}$, and focused on a $0.5 \mathrm{~mm}$ spot on the surface of the sample. The acquisition period for Raman spectra was $30 \mathrm{~s}$, with a $0.3 \mathrm{~cm}^{-1}$ spectral resolution over a $150-3400 \mathrm{~cm}^{-1}$ Raman shift range.

\subsection{Dataset}

A total dataset (D) of 267 Raman signals (10834 points) acquired using the protocol described before was finally obtained (see Figure 1. Each signal was manually labeled thanks to anatomical pathology analysis and affected to one or several predefined hierarchical groups as described in table 1.

\begin{tabular}{|c|c|c|c|}
\hline \multicolumn{4}{|c|}{ Total dataset (D): 267} \\
\hline \multirow{4}{*}{ Normal (N): 113} & \multicolumn{3}{|c|}{ Tumoral (T): 154} \\
\hline & \multirow{3}{*}{ Benign (B): 2} & \multicolumn{2}{|c|}{ Malignant (M): 152} \\
\hline & & Low-Grade (LG): 61 & High-Grade (HG): 91 \\
\hline & & Clear-Cell (CC): 117 & Other Types (OT): 35 \\
\hline
\end{tabular}

Table 1: Raman signals dataset and groups hierarchy description.

\section{Method}

\subsection{Overview}

To evaluate the interest of $\mathrm{RS}$ in kidney cancer, 4 main problems of supervised classification are addressed in this study. More precisely, in order 

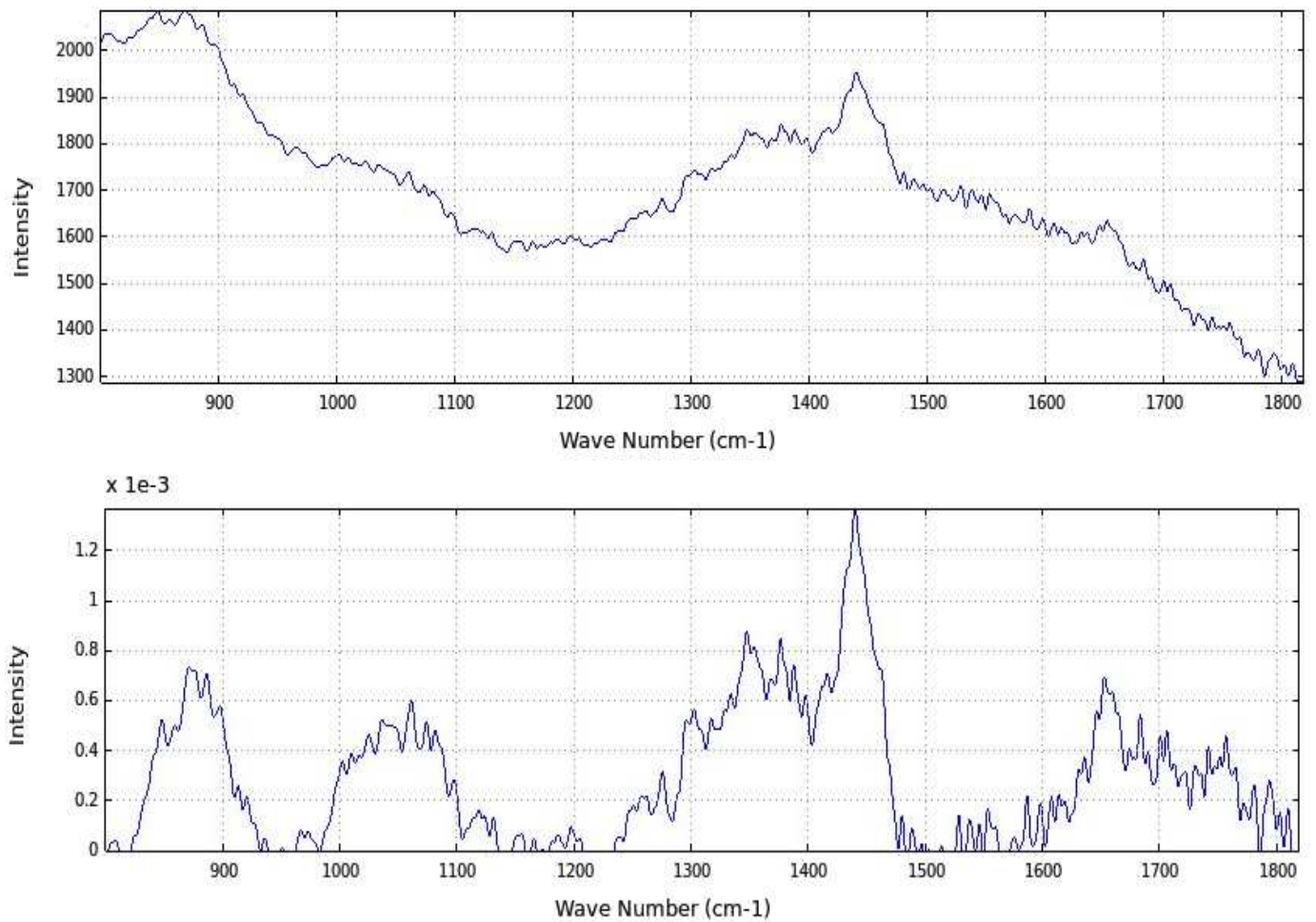

Figure 1: Example of raman spectra acquired on renal tissue: i) at the top, a raw renal spectrum and ii) at the bottom, a normalized spectrum after the autofluorescence background removal. 
to decide if the molecular information present in RS signal may be useful for the kidney cancer analysis, this study aims at evaluating the potential of RS to differentiate between: i) Normal Spectra (N) and Tumoural Spectra (T), ii) Low-Grade Spectra (LG) and High-Grade Spectra (HG), iii) Clear Cell Spectra (CC) and Other Types (OT), and iv) High-Grade Spectra (HG) and Not High-Grade Spectra (D - HG). Note that the other interesting problem of discrimination between malignant and benign spectra can not be addressed here because of the critical size of the benign group. Those 4 contexts of discrimination may obviously have a clinical interest, especially in a perspective of surgical assistance where the surgeon could have information about the tumour nature by a simple raman acquisition.

Each situation of renal spectra classification is handled by means of a common three-stage methodology involving a tunable preprocessing step, an appropriate supervised classifier, and a strategy to evaluate the quality of discrimination.

\subsection{Preprocessing}

Normalization. In a first time, to ensure comparability between spectra, a normalization step is performed by dividing each spectral point by the area of the total intensity of the spectrum [17].

Autofluorescence Background Removal. The kidney is of course highly vascularized and is mainly made of water as almost all the other organs. This high level of water therefore implies a very high level of autofluorescence in the raman signal that can mask the raman signal itself. This autofluorescence background is thus usually artificially removed from the original signal using 
multiple iterated polynomial regression [15] or PCA to identify dominant non Raman-signal [14] for example. Nevertheless, removing this background may be critical for two reasons: i) as the autofluorescence background can not be physically modeled, artificially removing the background with empirical techniques may induce errors in the spectra which could bias the classification scores and, ii) the autofluorescence background itself may contain some information which could be useful for the classification step. Two preprocessing strategies are therefore considered here: i) one strategy with no background removal, ii) another where the background is removed using a fifth order Modified Multi-Polynomial Fitting [15].

Spectral Band Selection. Studies from the literature generally only consider a sub-band of the whole spectrum for the analysis which is typically enclosed between 800 and $1600 \mathrm{~cm}^{-1}$ [16]. However the Raman spectroscopic system used during the acquisition gives access to a larger spectral band $150-3400 \mathrm{~cm}^{-1}$. To take advantage of this latter point, three options are proposed for the spectral band selection stage: i) keep only the standard band $800-1600 \mathrm{~cm}^{-1}$, ii) keep only the second level band $1600-3400 \mathrm{~cm}^{-1}$ or iii) keep the total spectral band $150-3400 \mathrm{~cm}^{-1}$.

Multi-Scale Filtering. Finally, considering the huge size of the normalized signals (more than 10000 points), a lower resolution signal may be computed for each spectrum by the mean of a wavelet decomposition [18] to help the classification stage. Such a transform may also remove high-frequency components from the signal (and especially potential noise and artefacts), may allow for selecting certain signal sub-bands for the analysis, and lets the 
possibility to take advantage of the whole spectral extent at a lower temporal resolution. On the other hand, filtering may remove some important discriminant information. Two configurations are thus considered for this preprocessing step: i) one configuration with no filtering, ii) another one allowing wavelet filtering, using two possible wavelets, namely Daubechies 3 and 6 , and where the $i^{\text {th }}$ approximation ( $i$ possibly varying from 1 to 14 ) is preserved.

The preprocessing stage is thus a highly tunable step. The influence of each free parameter on the classification may be critical and will be studied in section 4 .

\subsection{Classifier}

The preprocessed spectra are then given to a supervised classifier for learning and testing. In this prospective study, a Support Vector Machine (SVM) associated to a SMO solver [19] has been chosen for the classification step. SVM is well known for its numerical efficiency as well as its good capacity of generalization. To avoid any hyper-parameter setup, a linear kernel is first used to learn the different groups and to elaborate a classification strategy.

\subsection{Evaluation}

To obtain a classification accuracy and thus, to evaluate the potential of $\mathrm{RS}$ in kidney cancer, a leave-one-out cross validation technique [17] is used: all spectra excepted one are used for the machine training, and the remaining spectrum is reserved for the testing step. The process is repeated with alternation of the testing spectrum for each signal of the population of 
interest. A cross-validation score of good classification, a sensitivity and a specificity of the classification model are thus finally obtain by considering each cross-validation step and make it possible to evaluate the performance of a classification strategy based on RS signals.

\section{Results}

In this section, the previous methodology is applied to solve the four problems of classification considered in the study and described at the beginning of section 3. Various relevant configurations of the preprocessing stage are especially tested to investigate how RS should be useful in the context of kidney cancer characterization and how a classification strategy could be optimized.

\subsection{Autofluorescence Background Removal}

In this first part the influence of fluorescence background removal is studied. Two configurations of the classification procedure are compared and described in table 2. In a first test, the classification procedure is launched on the standard spectral band comprised between 800 and $1600 \mathrm{~cm}^{-1}$ with no background removal and no wavelet filtering. In the second configuration, the background is artificially removed with a fifth order Modified MultiPolynomial Fitting algorithm [15].

Results of both tests are described in table 3. For each context of classification, better results in terms of score, sensitivity and specificity are observed when the background is not removed. The shape of the autofluorescence background seems therefore to clearly influence the classification performances. On the contrary, a classification strategy only based on the 


\begin{tabular}{|c||c|c|}
\hline Configuration & $\# 1$ & $\# 2$ \\
\hline \hline Spectral Band $\left(\mathrm{cm}^{-1}\right)$ & \multicolumn{2}{|c|}{$800-1600$} \\
\hline Background Removal & No & Yes \\
\hline Wavelet filtering & \multicolumn{2}{|c|}{ No } \\
\hline
\end{tabular}

Table 2: Influence of the fluorescence background removal: Preprocessing setup description for the different scenarios.

"pure" raman signal (without any autofluorescence content) would not give satisfying result in the context of our study, except perhaps for the characterization of clear cell tumours. Keeping the background content in the context of RS kidney tumour analysis appears therefore as a critical point for a discriminant analysis. The important quantity of water in the kidney or the vascularization degree variation between the different classes of kidney tissues may explain those differences.

\begin{tabular}{|c|c|c||c|c||c|c||c|c|}
\hline Problem & \multicolumn{2}{|c||}{ N/T } & \multicolumn{2}{c||}{ LG/HG } & \multicolumn{2}{c||}{ CC/OT } & \multicolumn{2}{c|}{ HG/D-HG } \\
\hline Configuration & $\# 1$ & $\# 2$ & $\# 1$ & $\# 2$ & $\# 1$ & $\# 2$ & $\# 1$ & $\# 2$ \\
\hline Score (\%) & 77.53 & 65.92 & 77.63 & 64.47 & 89.47 & 81.58 & 81.27 & 71.54 \\
\hline Sensitivity (\%) & 76.11 & 63.72 & 80.22 & 65.93 & 92.31 & 86.32 & 86.36 & 79.55 \\
\hline Specificity (\%) & 78.57 & 67.53 & 73.77 & 62.30 & 80.00 & 65.71 & 71.43 & 56.04 \\
\hline
\end{tabular}

Table 3: Influence of the fluorescence background removal: Results.

\subsection{Band Selection}

Another point that we would like to consider in this study concerns the choice of an optimal spectral band of interest for a discriminant analysis. To 
evaluate the influence of this parameter, three scenarios with three different preprocessing configurations are compared and described in table 4. In each case a different spectral band is used for the classification procedure (the last scenario involves the complete spectral band).

\begin{tabular}{|c||c|c|c|}
\hline Configuration & $\# 1$ & $\# 2$ & $\# 3$ \\
\hline \hline Spectral Band $\left(\mathrm{cm}^{-1}\right)$ & $800-1600$ & $1600-3400$ & $150-3400$ \\
\hline Background Removal & \multicolumn{3}{|c|}{ No } \\
\hline Wavelet filtering & \multicolumn{3}{|c|}{ No } \\
\hline
\end{tabular}

Table 4: Influence of the spectral band selection: Preprocessing setup description for the different scenarios.

Results of both simulations are presented in tables 5 and 6 . The standard band comprised between 800 and $1600 \mathrm{~cm}^{-1}$ seems to be more interesting than the second band in almost all classification problems except for the specific case of discrimination between low-grade and high-grade tumours where the second band (comprised between 1600 and $3400 \mathrm{~cm}^{-1}$ ) could have a practical interest. Nevertheless, it clearly appears that taking advantage of the whole spectral content remains the best alternative to efficiently separate the different classes of renal tissue with the proposed methodology. In that case, very encouraging results are obtained to discriminate all kind of tissues with a lowest score of classification of almost $80 \%$ for the classification of normal vs. tumoral tissues. Very interesting scores are even obtained to recognize clear cell tumours from the other types of cancer (around $94 \%$ of good classification). In most cases, one can also observe good specificity and sensitivity values which highlight the nice behaviour of the proposed classifier. One can 
nevertheless observe the quite unbalanced behaviour between sensitivity and specificity when trying to differentiate high-grade and "not high-grade" tissues. However, from a general point of view, those results clearly demonstrate the potential of RS to analyze renal tumoral tissues.

\begin{tabular}{|c|c|c|c||c|c|c|}
\hline Problem & \multicolumn{3}{|c||}{ N/T } & \multicolumn{3}{c|}{ LG/HG } \\
\hline Configuration & $\# 1$ & $\# 2$ & $\# 3$ & $\# 1$ & $\# 2$ & $\# 3$ \\
\hline Score (\%) & 77.53 & 73.41 & 79.78 & 77.63 & 81.58 & 83.55 \\
\hline Sensitivity (\%) & 76.11 & 74.34 & 79.65 & 80.22 & 82.42 & 85.71 \\
\hline Specificity (\%) & 78.57 & 72.73 & 79.87 & 73.77 & 80.33 & 80.33 \\
\hline
\end{tabular}

Table 5: Influence of the spectral band selection: Results (Part 1).

\begin{tabular}{|c|c|c|c||c|c|c|}
\hline Problem & \multicolumn{3}{|c||}{ CC/OT } & \multicolumn{3}{c|}{ HG/D-HG } \\
\hline Configuration & $\# 1$ & $\# 2$ & $\# 3$ & $\# 1$ & $\# 2$ & $\# 3$ \\
\hline Score (\%) & 89.47 & 84.21 & 94.74 & 81.27 & 79.78 & 82.40 \\
\hline Sensitivity (\%) & 92.31 & 85.47 & 95.73 & 86.36 & 85.80 & 87.50 \\
\hline Specificity (\%) & 80.00 & 80.00 & 91.43 & 71.43 & 68.13 & 72.53 \\
\hline
\end{tabular}

Table 6: Influence of the spectral band selection: Results (Part 2).

\subsection{Influence of Wavelet Filtering}

In this last section, the influence of wavelet filtering on the discrimination capabilities of raman signals is studied. As previously mentioned in section 3, a wavelet transform may help for a better classification by reducing the signal size and filtering the spectral content. To study the interest of such a 
transform on the classification scores, a wavelet transform is applied to each raman spectra during the preprocessing step. Two different wavelets are used and several levels of decomposition are tested as described in table 7 .

\begin{tabular}{|c||c|c|}
\hline Configuration & $\# 1$ & $\# 2$ \\
\hline \hline Spectral Band $\left(\mathrm{cm}^{-1}\right)$ & \multicolumn{2}{|c|}{$150-3400$} \\
\hline Background Removal & \multicolumn{2}{|c|}{ No } \\
\hline Wavelet filtering & Daubechies 3 & Daubechies 6 \\
\hline
\end{tabular}

Table 7: Influence of a wavelet transform: Preprocessing setup description for the different scenarios.

Figure 2 presents the influence of this preprocessing step on the classification score for the four classification problems. More finely, for each scenario, the classification score obtained after a wavelet transform with the two considered wavelets is presented with respect to the level of decomposition. Several remarks can be pointed out when analyzing figure 2. First, using a lower resolution of the signal may really improve the classification results, especially when discriminating normal and tumoral spectra: an improvement of $5 \%$ of good classification can be observed when using the $8^{\text {th }}$ approximation. In such a case, the low-frequency content of the signal seems to be the most discriminant and confirms the fact that the low-frequency autofluorescence background has an important role in the classification procedure. Moreover, in the other classification situations (High-Grade vs Low-Grade, Clear Cell vs. Other Types and High-Grade vs. Not High Grade), using a lower resolution (until level 4) may not really affect the classification score: an instrument with a lower precision and thus, with a lower cost, could therefore 

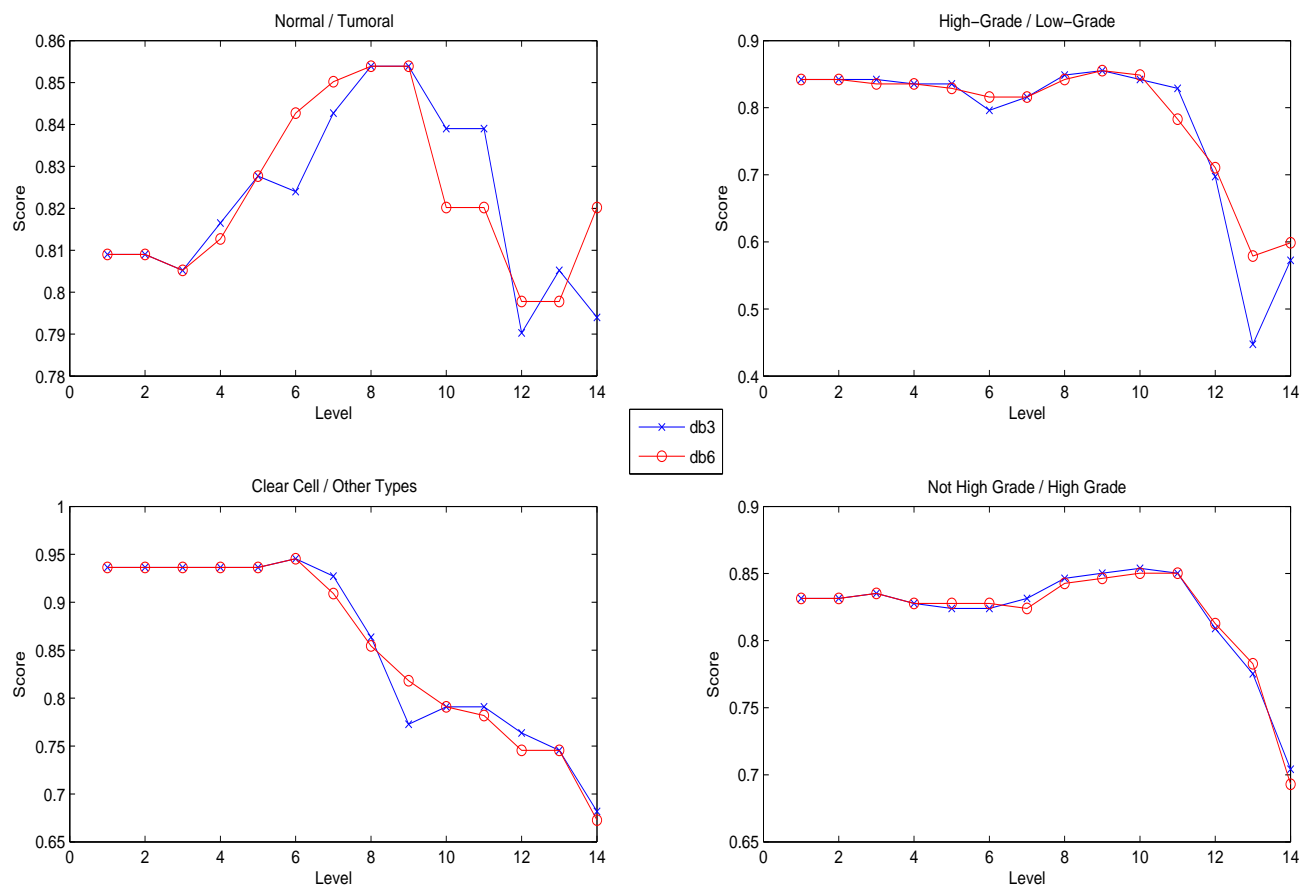

Figure 2: Influence of a wavelet transform: Classification score after a Daubechies 3 (circle) and a Daubechies 6 (cross) wavelet transform with respect to different level of decomposition (1 to 14$)$

be used successfully to realize the same task. Finally, no concrete difference can be really found in the classification score between a Daubechies 3 or 6 wavelet transforms. The shape of the wavelet seems therefore not to have a determinant role in such a preprocessing step. 


\section{Conclusion and Perspectives}

In conclusion, in this preliminary study, a classification procedure based on a SVM classifier has been proposed to show how RS could robustly differentiate various classes of renal and tumoural tissues. The study has especially shown how RS could efficiently discriminate between normal and tumoural tissues but also, how RS could even help to identify various grades and types of cancer. Moreover, this work has highlighted how the fluorescence background as well as a larger spectral band of analysis could be used to improve the classification score in the context of renal spectral discrimination. The combination of RS with the proposed tunable classification procedure could therefore be pictured as an "optical biopsy tool" that has several possible applications in clinical urology and that could help the pathologist to improve diagnostic accuracy.

However, several points still remain to be improved in the future. First, the study was done on ex-vivo specimen, and the impact of in-vivo parameters might have been underestimated. For this reason, there is a need for in vivo studies with, of course, a refinement of the protocol and of the equipment to be used in the sterile environment of the operating room. Second, our population of benign lesions was too limited to demonstrate any difference between benign and malignant renal tumours and larger studies need now to be undertaken to correlate optical profiles to the outcome of kidney cancer. Finally, and concerning the classification methodology itself, a deep analysis of several points can be now conducted (configuration of the support vector machine with an alternative kernel choice for example) to optimize the efficiency of the classification. 


\section{Acknowledgements}

This work was supported by the National Research Agengy (ANR) of France under Grant DIAPRECA ANR-06-TecSan-013-01 and ANR-06-TecSan013-03.

[1] W.H. CHOW and S.S. DEVESA, "Contemporary epidemiology of renal cell cancer," Cancer J, vol. 14, pp. 288-301, 2008.

[2] A. RAVAUD, H. WALLERAND, S. CULINE et al, "Update on the medical treatment of metastatic renal cell carcinoma," Eur Urol, vol. 54, pp. 315-325, 2008.

[3] J.S. LAM, J.T. LEPPERT, R.A. FIGLIN et al, "Role of molecular markers in the diagnosis and therapy of renal cell carcinoma," Urology, vol. 66, pp. 1-9, 2005.

[4] G.P. GUPTA, L. MASSAGUE, "Cancer metastasis: building a framework," Cell, vol. 127, pp. 679-695, 2006.

[5] D. HANAHAN, R.A. WEINBERG, "The hallmarks of cancer," Cell, vol. 100, pp. 57-70, 2000.

[6] D.J. PAREKH, W.C. LIN, S.D. HERRELL, "Optical spectroscopy characteristics can differentiate benign and malignant renal tissues: a potentially useful modality," The Journal of urology, vol. 174, pp. 1754-1758, 2005.

[7] K. BENSALAH, A. TUNCEL, D. PESHWANI et al, "Optical reflectance 
spectroscopy to differentiate renal tumor from normal parenchyma," The Journal of urology, vol. 179, pp. 2010-2013, 2008.

[8] K. BENSALAH, D. PESWANI, A. TUNCEL et al, "Optical reflectance spectroscopy to differentiate benign from malignant renal tumors at surgery," Urology, vol. 73, pp. 178-181, 2009.

[9] P. Crow, A. Molckovsky, N. Stone et al, "Assessment of fiberoptic nearinfrared raman spectroscopy for diagnosis of bladder and prostate cancer," Urology, vol. 65, pp. 1126-1130, 2005.

[10] N. STONE, M.C. HART PRIETO, P. CROW et al, "The use of Raman spectroscopy to provide an estimation of the gross biochemistry associated with urological pathologies," Analytical and bioanalytical chemistry, vol. 387, pp. 1657-1668, 2007.

[11] L. LOVAT, S. BOWN, "Elastic scattering spectroscopy for detection of dysplasia in Barrett's esophagus," Gastrointestinal endoscopy clinics of North America, vol. 14, n. 9, pp. 507-517, 2004.

[12] P. CROW, J.S. UFF, J.A. FARMER et al, "The use of Raman spectroscopy to identify and characterize transitional cell carcinoma in vitro," BJU international, vol. 93, pp. 1232-1236, 2004.

[13] P. CROW P, N. STONE, C.A. KENDALL et al, "The use of Raman spectroscopy to identify and grade prostatic adenocarcinoma in vitro," British journal of cancer, vol. 89, pp. 106-108, 2003.

[14] J.V. GLENN, J.R. BEATTIE, L. BARRETT, N. FRIZZELL, S.R. 
THORPE, M.E BOULTON, J.J. MCGARVEY and A.W. STITT, "Confocal Raman microscopy can quantify advanced glycation end product (AGE) modifications in Bruch's membrane leading to accurate, nondestructive prediction of ocular aging," FASEB J., vol. 21, n. 13, pp. 3542$3552,2007$.

[15] J. ZHAO, H. LUI, D.I. MCLEAN and H. ZENG, "Automated Autofluorescence Background Subtraction Algorithm for Biomedical Raman Spectroscopy," Appl. Spectrosc., vol. 61, pp. 1225-1232, 2007.

[16] E.B. HANLON, R. MANOHARAN, T.W KOO, K.E. SHAFER, J.T. MOTZ, M. FITZMAURICE, J.R. KRAMER, I. ITZKAN, R.R. DASARI and M.S. FELD, "Prospects for in vivo Raman spectroscopy," Phys. Med. Biol., vol. 45, pp. R1-R59, 2000.

[17] S. BUM KIM , C. TEMIYASATHIT, K. BENSAlAH, A. TUNCEL, J. CADEDDU, W. KABBANI, A.V. MATHKER and H. LIU, "An effective classification procedure for diagnosis of prostate cancer in near infrared spectra", Expert Systems with Applications, vol. 37, pp. 38633869, 2010.

[18] S. MALLAT, "A Wavelet Tour of Signal Processing, Third Edition: The Sparse Way", Academic Press, 2008.

[19] J.C. PLATT, "Fast training of support vector machines using sequential minimal optimization in Advances in kernel methods: support vector learning", MIT Press, pp. 185-208, 1999. 